\title{
Lumen
}

Selected Proceedings from the Canadian Society for Eighteenth-Century Studies

\section{Surnoms et écriture codée dans les lettres de Mme de Graffigny}

\section{Diane Beelen Woody}

Volume 37, 2018

URI : https://id.erudit.org/iderudit/1042226ar

DOI : https://doi.org/10.7202/1042226ar

Aller au sommaire du numéro

Éditeur(s)

Canadian Society for Eighteenth-Century Studies / Société canadienne d'étude du dix-huitième siècle

ISSN

1209-3696 (imprimé)

1927-8284 (numérique)

Découvrir la revue

Citer cet article

Beelen Woody, D. (2018). Surnoms et écriture codée dans les lettres de Mme de Graffigny. Lumen, 37, 107-121. https://doi.org/10.7202/1042226ar d'utilisation que vous pouvez consulter en ligne.

https://apropos.erudit.org/fr/usagers/politique-dutilisation/ 


\title{
Surnoms et écriture codée dans les lettres de Mme de Graffigny
}

\author{
Diane Beelen Woody \\ York University
}

Quinze volumes de la Correspondance de Mme de Graffignyl ont maintenant été publiés, provenant de la période entre 1738 et 1758 , année de son décès. Elle offre une chronique fascinante de ces deux décennies du règne de Louis XV, lorsque les termes «secret» et «surveillance» s'employaient fréquemment. Car durant ces années ont eu lieu des événements politiques et sociaux d'importance majeure tels la guerre de Succession d'Autriche, la crise de 1744 lorsque Louis $\mathrm{XV}$ est tombé gravement malade à Metz, les émeutes de 1750 à Paris, l'attentat de Damiens contre le roi en 1757 et les débuts de la guerre de Sept Ans. De tels remous et conflits offraient aux autorités les justifications d'une surveillance accrue pour des «raisons d'État». En fait, on trouve chez plusieurs chroniqueurs (par exemple, Barbier et Luynes$^{2}$ ) des allusions à l'ouverture des lettres privées, au Secret de la Poste et à la création du Secret du Roi par Louis XV. À cet égard,

1. Correspondance de Mme de Graffigny, éd. de J.A. Dainard et E. Showalter, Oxford, Voltaire Foundation, 1985-2016, 15 vol. Les citations renvoient à cette édition, qui reproduit l'orthographe de Mme de Graffigny. Désormais, nous précisons le volume et la page, ainsi que le numéro et la date de la lettre citée entre parenthèses. Nous tenons à remercier Dorothy P. Arthur, English Showalter et David W. Smith qui ont lu une version préliminaire de cet article.

2. Voir Edmond Jean François Barbier, Chronique de la régence et du règne de Louis XV, Paris, Charpentier, 1857, vol. VIII, p. 183-184, au sujet de la "visite des lettres » dans le «Journal de police » d'octobre 1742 ; et Charles-Philippe d'Albert, duc de Luynes, Mémoires sur la cour de Louis XV (1735-1758), Paris, Firmin Didot frères et fils, 1860-1865, au sujet des lettres décachetées, vol. IX, p. 243 (octobre 1748) et vol. XII, p. 100 (août 1752). 
l'ouvrage d'Eugène Vaillé fournit une discussion approfondie des activités du Cabinet noir et celui de Jay Caplan ${ }^{4}$ offre un examen détaillé de la «culture postale» en Europe avant 1800 et une analyse de l'enchevêtrement des domaines public et privé. On s'attend donc à trouver des reflets de cette réalité de surveillance dans les lettres de Mme de Graffigny, qui devait gérer stratégiquement ses relations avec ceux qu'elle appelait «les grands » pour obtenir une pension royale et pour protéger sa propre réputation d'auteure distinguée acquise à la suite de ses succès littéraires.

Nous proposons d'examiner la situation de Mme de Graffigny, la surveillance à laquelle elle était sujette, ses inquiétudes, son recours à l'écriture codée et son utilisation de surnoms dans les lettres écrites à son correspondant principal, François-Antoine Devaux. Sans prétendre à une étude exhaustive, nous envisageons d'examiner les surnoms utilisés dans la Correspondance, d'identifier une typologie et de dégager quelques principes qui gouvernent leur création et les diverses motivations derrière leur emploi, dans le but de mieux comprendre la relation entre ces surnoms et l'écriture codée.

Mme de Graffigny comptait parmi ses connaissances un grand nombre de personnalités éminentes de l'époque, y compris d'illustres représentants de différentes cours d'Europe. Elle connaissait bien les membres des familles lorraines Beauvau-Craon et La Galaizière. Elle écrivait de petites pièces pour les enfants de la cour de Vienne et a dédié sa dernière pièce à l'impératrice Marie-Thérèse d'Autriche (voir 15: 332, lettre 2492, fin octobre 1758). Pour Anne-Charlotte, princesse de Lorraine à la cour de Vienne, elle faisait des commissions, parmi lesquelles on compte celle de faire peindre le portrait de Mme de Pompadour (8: 125, lettre 1073, 6 novembre 1746). La maîtresse du roi voulait aussi que Mme de Graffigny se charge de l'éducation de sa fille, Alexandrine Lenormant d'Étiolles (10: 147, lettre 1437, 11 août 1749). Après le succès de son roman Lettres d'une Péruvienne et celui de sa pièce Cénie, Mme de Graffigny était devenue elle-même une «illustre»; La Tour voulait faire son portrait (11: 284, lettre 1639, 11 décembre 1750) et la margrave de Brandebourg-Bayreuth, Sophie-

3. Eugène Vaillé, Le cabinet noir, Paris, Presses universitaires de France, 1950.

4. Jay Caplan, Postal Culture in Europe, 1500-180o, Oxford, Voltaire Foundation, 2016; voir en particulier p. 101-118. 
Frédérique-Wilhelmine, sœur de Frédéric II, voulait l'attirer à sa cour (11: 110, lettre 1593, 26 août 1750).

Dans le milieu politique et militaire, Mme de Graffigny entretenait des relations avec le comte de Clermont, le prince Charles-Alexandre de Lorraine et les membres de la famille d'Argenson. Notons que deux amis, l'ambassadeur Starhemberg et l'abbé de Bernis, avaient participé aux négociations en 1756 et 1757 du fameux «renversement des alliances », c'est-à-dire la révolution diplomatique qui a vu la France s'allier avec l'Autriche en signant deux traités à Versailles. Lors de l'insuccès de sa dernière pièce La fille d'Aristide, Mme de Graffigny explique à Devaux que ses «ennemis» devraient être contents de l'échec et elle ajoute qu'elle a la réputation d'avoir joué un rôle dans la renégociation des alliances: «Tu ne devinerois pas un des motifs de leur acharnement: c'est que je n'ai pas peu contribué au traité d'union » (15:226, lettre 2427,7 mai 1758). On remarquera aussi qu'en 1757, elle a reçu des lettres écrites de différents camps militaires (15: lettre 2402 de François de Chevert, lettre 2413 de Louis-François de Civalard, et les lettres 2412, 2414, 2417 et 2419 écrites par un ami militaire [non identifié]). Enfin, son ami le comte de Maillebois, Yves-Marie Desmarets, lui a écrit de la citadelle de Doullens où il avait été emprisonné lors de sa disgrâce en 1758 (15: 236, lettre 2433, 26 mai 1756).

Cet impressionnant réseau de connaissances incluait des personnes auxquelles les autorités auraient pu s'intéresser. En fait, tout au long de la Correspondance, on trouve des indices de la surveillance ainsi que de la peur des conséquences néfastes qui pouvaient résulter d’un manque de prudence. Il y a de nombreuses allusions aux deux lieutenants de police entre 1740 et 1757 (Feydeau de Marville et NicolasRené Berryer) et aux mouches (par exemple, le chevalier de Mouhy). Il importe de noter aussi que le comte d'Argenson, que Mme de Graffigny connaissait bien, était surintendant général des postes entre 1744 et 1757 et que sous son administration, on ouvrait, lisait et recachetait certaines lettres privées. On trouve dans la Correspondance de nombreuses allusions aux prisons (par exemple, la Bastille, Bicêtre, For-l'Évêque et Vincennes) et aux personnes qui y étaient détenues, dont des auteurs (Basset Des Rosiers, Diderot, Fréron, La Beaumelle et l'abbé de Prades) et des acteurs (Armand, La Noue et même Lekain). La liste n'exclut pas des personnes qu'on peut caractériser comme membres du cercle intime de Mme de Graffigny, par exemple, Antoine 
Bret (emprisonné à Vincennes en 1749) et Léopold Liébault (détenu à la Bastille, puis à Vincennes en 1758).

Enfin il y a de nombreuses mentions explicites de l'ouverture et de l'interception de lettres. En 1738, Mme de Graffigny avertit Devaux: «A propos, regarde bien les letres en les ouvrant, pour voir s'il n'y a rien au cachet; j’ai peur. Et averti-moi s'il y a quelque chose» (1:212, lettre 62, 11 décembre 1738). En 1749, lors de la disgrâce de Maurepas, notre épistolière fait mention des chansons et épigrammes contre la Marquise de Pompadour : «Je crois bien que personne n’a eté assés insensé pour les envoier en province qu'un abbé [non identifié] qui a eté mis a la Bastille qui les envoioit tout bonnement dans une lettre signée. Tant que cela a duré, toutes les letres ont eté ouvertes » (10:22, lettre 1396, 8 mai 1749). La situation perdure et en 1750, Mme de Graffigny écrit: «Le chevalier de Resseguier est a la Bastille pour des vers interceptés dans une letre» (11: 294, lettre 1641, 13 décembre 1750). Mme de Graffigny a formulé un principe de base qu'elle explique à Devaux: «Tu es bien fou de me demander des nouvelles de Mde Sauvé [femme de chambre du duc de Bourgogne]. Est-ce que je me mele des affaires des grands? On ouvre les letre dans de pareil cas. Si une fois on avoit trouvé de pareilles nouvelles, on les ouvriroit toujours. Je ne veux jamais me metre dans cet embaras » (12: 158, lettre 1781, 3 novembre 1751).

Les inquiétudes principales étaient l'ouverture et la perte de lettres car une lettre perdue aurait pu être interceptée ou volée:

Enfin voila donc decidé qu'il y a une de tes lettres perduë. [...] Je crains que tu ne sois entré dans de trop grands details sur notre perte, sur mes affaires. Je suis sure que cette lettre devieroit quelq'un a Versailles. Je vis hier Disenteuil [l'abbé de La Galaizière], qui n'en avoit aussi recu aucune de Luneville, mais plusieurs de divers endroits de la Lorraine. Je presume, et lui aussi, que l'on aura porté a la cour le paquet de Luneville pour etre visité. Eh mon Dieu, notre pauvre petite conversation n'est pas faite pour etre en tier avec de si grands personnage [sic]: nous n'avons que des secrets de sentimens a nous communiquer $(7: 132$, lettre 932, 14 décembre 1745).

Bien qu'on trouve de tels passages où Mme de Graffigny exprime ouvertement ses peurs et ses inquiétudes, de manière générale, on ne peut pas parler d'un climat généralisé d'affolement et d'angoisse dans les lettres. Le projet de Mme de Graffigny était d'informer son correspondant Devaux des nouvelles de la cour, de la vie parisienne et de 
tout ce qui avait rapport au monde des lettres, y compris le théâtre. Le défi était de le faire en pleine compréhension des enjeux, comme elle le rappelle à Devaux en abordant le sujet des lettres ouvertes: «Grosse bete, est que Richard [François Richard, directeur de la poste aux lettres de Lunéville] ira chaque fois demander au roi si c'est par son ordre quand une fois l'homme de confiance est designé? [...] Mais toi qui aime tant les histoires qui developent les intrigue de cour, les friponnerie et les abus, tu les prends pour des contes de fées peut-etre? » (11: 557, lettre 1714, 30 mai 1751). Il importe de ne pas exagérer l'intérêt que pouvaient susciter les lettres de Mme de Graffigny et de Devaux, en Lorraine aussi bien qu'en France; cependant, les indices figurant dans les lettres nous montrent que des mesures de prévoyance s'imposaient ${ }^{5}$. Parmi celles-ci, on peut citer la pratique de confier des lettres à des particuliers qui faisaient le trajet entre la Lorraine et Paris, afin d'éviter le système de poste. Une autre stratégie était celle de l'écriture codée pour éviter de nommer explicitement des personnes ou des événements qui pourraient retenir l'attention en cas d'ouverture des lettres. Le recours aux surnoms peut se voir comme un aspect de cet encodage.

Mme de Graffigny et Devaux utilisent très fréquemment dans leurs lettres des surnoms pour désigner des personnes dont ils discutent. Au début de la Correspondance, on trouve même une allusion explicite à une clef des surnoms, une sorte de légende: «Tu as bien fait de m’envoyer la clef » $(1: 218$, lettre 63,13 décembre 1738$)$ et plus loin dans la même lettre: "Ajoute a ta clef Nicodeme pour V. [Voltaire] et Dorotée pour sa bergere [Mme Du Châtelet], car je crains pour ici et moiennant cela, je pourai dire bien des choses que je ne peus pas » (1:224). Une réponse de Devaux confirme que c'est par prévoyance qu'on changeait de surnoms: «Avez-vous mandé a Dorothée [Mme Du Châtelet] l'histoire du sac? Je crois que nous devrions changer ce nom. Si nos lettres etoient interceptées elle pourroit se reconnoitre» (1: 375 , lettre 102, voir note 62 pour le texte de Devaux). Une motivation majeure derrière l'emploi des surnoms semble être le désir de conserver la liberté de se dire «bien des choses» qu'on ne pourrait pas exprimer autrement.

5. Voir aussi English Showalter, «L'interdit dans la correspondance de Françoise de Graffigny", dans L'épistolarité à travers les siècles: geste de communication et/ou de l'écriture, Stuttgart, Steiner, 1990, p. 143-146. 
En l'absence de la clef ou légende mentionnée ci-dessus, la tâche des éditeurs de la Correspondance s'avère compliquée. Une consultation de l'index cumulatif en ligne de la Correspondance ${ }^{6}$ nous permet de constater que plus de 500 surnoms sont utilisés dans les lettres. Pour le décodage des surnoms, le contexte spécifique et les lettres de Devaux (que nous possédons jusqu'en juillet 1751) ont fourni une aide précieuse ainsi que de nombreuses sources classiques imprimées et des ressources d'archives ${ }^{7}$. Il faut avouer qu'à certains moments, le décodage proposé n'est que probabilité, et que dans une quinzaine de cas, les surnoms restent «non identifiés » ou «non décodés». Néanmoins, dans l'ensemble, le travail d'identification a pu se faire même plus de 250 ans après la rédaction de ces lettres. On serait tenté d'en conclure que les surnoms utilisés par Mme de Graffigny sont trop transparents et donc inefficaces comme écriture codée. Ce serait trop vite confondre l'écriture codée qui a pour but de brouiller les pistes et l'emploi de surnoms qui relève de toute une gamme de motivations.

Il est donc possible de distinguer quelques schémas qui permettent d'esquisser une taxonomie des surnoms offrant différents degrés de transparence. Une première catégorie est celle des surnoms créés à partir d'une lettre initiale. Ainsi on retrouve le «C.» [Clairon Lebrun], le «D. » [Desmarest], le «D.F. » ou «L.D.F.» [l'abbé Desfontaines], le «P.C.» [le prince Charles] et «V.»(Voltaire). De tels surnoms démontrent un haut degré de transparence, sont faciles à décoder et relèvent probablement d'un souci de concision. Par exemple, lorsque Mme de Graffigny commence à utiliser le surnom «Atis » pour désigner Voltaire, elle explique: «c'est le nom que je mets a la place de Nicodeme, parce qu'il est plus court» (I : 292, lettre 80, 19 janvier 1739).

Une deuxième catégorie est celle des surnoms basés sur une désignation par nationalité ou par un autre indice géographique, procédé qui ne surprend pas chez l'auteure des Lettres d'une Péruvienne. Ainsi retrouve-t-on des désignations comme «Ma Vieille Anglaise» [Mme Macarty], «le Dogue d'Angleterre » [Dromgold], «mon Italienne»

6. L'index cumulatif, qui donne aussi une liste de surnoms et une liste de locutions, est disponible sur le site de l'université de Toronto : http://french.chass.utoronto. ca/graffigny/g16/cumind/files/a.html.

7. À ce sujet, voir l'article de Marie-Thérèse Inguenaud, «Comment identifier les personnages d'une Correspondance? Utilisation des ressources d'Archives: l'exemple de Mme de Graffigny », Épistolaire. Revue de l'A.I.R.E., 33, 2007, p. 81-94. 
[Mlle Bagarotti], «le Lapon » [Maupertuis], « le Phénomène du Nord » [Frédéric II] et «le Suisse» [Koenig]. En général, ces surnoms, eux aussi, s'avèrent assez faciles à pénétrer, surtout que le contexte dans lequel apparaît la désignation fournit souvent d'autres détails sur la personne en question et parfois même le nom au complet. Le but de ces surnoms ne semble pas être de cacher l'identité des gens; il s'agit peut-être de varier la formulation vu que, pour la plupart, les personnes ainsi désignées sont nommées explicitement à d'autres moments. Remarquons toutefois qu'une «Anglaise», que Mme de Graffigny appelle «une très bonne amie» (12: 358, lettre 1864, 9 mai 1752) reste non identifiée.

Une troisième catégorie de surnoms est celle de la désignation par profession ou par rang social. Ainsi retrouve-t-on une surabondance d'abbés et de jésuites, de comtes/comtesses, de ducs/duchesses, de marquis/marquises et de princes/princesses. Dans l'ensemble, grâce aux détails fournis dans les lettres de Devaux et selon le contexte des lettres individuelles, on a pu identifier les individus ainsi désignés; il faut cependant noter qu'à certains moments, les éditeurs de la Correspondance n'ont pas pu effectuer une identification certaine et proposent au lecteur plusieurs noms possibles. Un aspect qui mérite d'être souligné est que de tels termes, surtout dans le cas des désignations par rang social, sont souvent précédés d'une marque de politesse, par exemple «Monsieur le Duc» [Louis-Henri, duc de Bourbon] ou - ce qui est très fréquemment le cas - d'un adjectif tel «beau/belle» ou «petit/petite». Quelques exemples en sont «la Belle Duchesse» [la duchesse de Châteauroux], «la Belle Princesse» [la princesse AnneCharlotte], «la Belle Marquise» [la marquise de Boufflers/la marquise de Pompadour], «le Beau Ministre » [d'Argenson], «le Petit Marquis » [Amezaga], et «le Petit Ministre» [Saint-Florentin]. Par moments, de tels adjectifs deviennent plus descriptifs et même superlatifs, par exemple «le Prince Protecteur» [le comte de Clermont], «le Prince Charmant» [Beauvau-Craon], «la Très Belle » et «la Belle des Belles » [Anne-Charlotte de Lorraine]. De tels surnoms ne sont pas suffisamment opaques pour dérouter les espions; leur transparence relative invite donc à formuler d'autres hypothèses.

Mme de Graffigny et Devaux s'écrivaient à une époque où se publiait un grand nombre de mémoires, de pseudo-mémoires, de journaux et d'écrits autobiographiques où fiction et réalité s'entremêlaient. Par exemple, deux ouvrages discutés dans ces lettres portent les 
titres Mémoires de Mme la Comtesse de $M^{* * * * *}$ (de la comtesse de Murat de Castelnau) et Mémoires de Monsieur le marquis de St ${ }^{\text {***; }}$ ou les amours fugitifs du cloître (du marquis d'Argens). En fait, l'index cumulatif de la Correspondance indique des centaines de titres qui débutent par les mots « Mémoires, Lettres, Histoire ou Vie». Bien entendu, ces titres à décoder avaient plusieurs motivations, dont la nécessité d'agir par prévoyance en supprimant les noms de personnes réelles, mais aussi le désir d'intéresser le public et d'attiser les lecteurs. Il est permis de parler d'une motivation mixte où prévoyance, protocole et pratique courante se confondaient. À ce sujet, on peut citer Françoise Weil qui, en analysant la censure, parle d'une zone floue entre l'autorisé et l'interdit: "Les auteurs et les éditeurs savaient très bien ce qui n'avait aucune chance d'être autorisé et ce qui avait quelque chance de passer entre les mailles, autrement dit, ce qui risquait d'être toléré ${ }^{8}$.» La pratique d'une sorte d'autocensure assurait que l'écrit soit acceptable tout en conservant une signification claire. L'emploi des surnoms par Mme de Graffigny se situe dans le droit fil des conventions ou protocoles de l'époque, et montre qu'elle ne se distingue pas par son audace afin de se protéger en cas d'ouverture de ses lettres. L'emploi des adjectifs mélioratifs déjà remarqués sert à renforcer l'idée que l'emploi de ces surnoms n'a au fond rien d'offensif ou de blâmable. Du moins, notre épistolière pourra, au besoin, raisonnablement désavouer l'intention d'offenser autrui et les autorités, pour leur part, pourront aussi s'expliquer s'ils n'ont rien trouvé de censurable après avoir ouvert et parcouru une de ses lettres.

Une quatrième catégorie de surnoms démontre l'une des techniques d'invention préférées de Mme de Graffigny. C'est le recours à des allusions littéraires, qui sont d'ailleurs très fréquentes dans la Correspondance et qui rendent ces lettres extrêmement agréables à lire aujourd'hui. Par exemple, un auteur peut être désigné par son personnage; ainsi Montesquieu sera «le Persan» et «Usbek»; Prévost sera «Des Grieux ». Pour d'autres surnoms, Mme de Graffigny puise dans la littérature dont raffolent les deux correspondants. On trouve de nombreuses allusions aux pièces de théâtre et aux personnages:

8. Françoise Weil, «Le fonctionnement de la censure en France au XVIII ${ }^{\mathrm{e}}$ siècle », dans Jacques Domenech (éd.), Censure, autocensure et art d'écrire: De l'Antiquité à nos jours, Bruxelles, Éditions complexe, 2005, p. 128. 
«L'amour précepteur» [l'abbé de Bernis], «Le diable boiteux» [la Barolière/le comte de Croix], «Harpagon» [le comte de Mareil], «le Métromane » [Turgot], «Turcaret» [Brassac] et «Tartuffe» [Grandville] pour n'en citer que quelques-uns. D’autres personnages viennent des romans : «Artamène » de Mlle de Scudéry [pour désigner La Barolière], «Don Sanche» et «Dulcinée» de Cervantes [pour désigner Stanislas et Mlle de Brun], et «Lovelace» de Richardson [pour désigner Antoine Chaumont de La Galaizière]. Ces surnoms riches en pouvoir évocateur sont des indices de ce qu'on appelle la «République des lettres», terme qu'on retrouve sous la plume de Mme de Graffigny elle-même (15: 265, lettre 2453, 12 juillet 1758). Lexpression évoque les personnes d'esprit et d'intelligence, une société de gens cultivés qui savouraient de telles allusions et qui étaient à l'affût de vérités cachées9. Un sujet d'intérêt particulier à l'époque était la vie privée du roi et d'autres membres de la cour. Mme de Graffigny mentionne explicitement Tanastès, le conte allégorique écrit par Mlle de Bonafons (6: 494, lettre 877,25 juillet 1745) et aussi la rumeur en 1752 de «La Vie scandaleuse de la cour et de Paris, ou tout le monde est nommé » en 6 volumes, bien que le titre soit non attesté (12: 255, lettre 1823, 4 février 1752). Un autre exemple serait le pamphlet Idée de la personne, de la manière de vivre et de la cour du roy de Prusse (13: 269, lettre 2016, 2 mai 1753). Ces titres renforcent la notion de la lecture au XVIII ${ }^{\mathrm{e}}$ siècle comme acte de décodage: lire signifiait en effet la capacité de décrypter à plusieurs niveaux.

Étant donné leurs ambitions et projets littéraires, Mme de Graffigny et Devaux participaient à cette culture. Ils étaient bien placés pour l'apprécier et pour y contribuer à leur tour par des inventions créatives. Comme le signale Aurora Wolfgang, «toute lettre, authentique ou fictive, passe par l'intermédiaire d'une construction littéraire ${ }^{10} »$, et on constate chez nos deux correspondants une conscience aiguë de leurs prédécesseurs. En mai 1739, Mme de Graffigny écrira à Devaux au sujet d'une de ses lettres: «C'est un tissus [sic] de Sevigné rebroché de

9. Voir, par exemple, Dena Goodman, The Republic of Letters: A Cultural History of the French Enlightenment, Ithaca (NY), Cornell University Press, 1994, et Colin Jones, The Great Nation: France from Louis XV to Napoleon, Londres, Penguin, 2002, p. 180.

10. Aurora Wolfgang, "Notre vieille amie" : Mme de Sévigné dans la correspondance de Mme de Graffigny et Devaux ", Studies on Voltaire and the Eighteenth Century, 12, 2004, p. 117. 
marivaudage » $\left(1: 487\right.$, lettre 129, 12 mai 1739) ${ }^{11}$. Selon cette perspective, l'emploi des surnoms permet aux deux correspondants de créer ce qui ressemble à un «langage privél2 ». Comme le fait remarquer Elizabeth MacArthur à propos de la correspondance de Mme du Deffand, les surnoms constituent des motifs qui créent un univers textuel relativement fermé13. Ceux et celles qui écrivent des lettres sont inévitablement menés à structurer consciemment leur correspondance pour suivre les conventions épistolaires et aussi pour anticiper d'autres publics, vu que très souvent les lettres étaient lues à haute voix ${ }^{14}$. Tout comme chez Mme du Deffand, dans les lettres de Mme de Graffigny, les surnoms constituent une stratégie rhétorique qui facilite la création de personnages ${ }^{15}$ et la re-structuration de la vie quotidienne selon des schémas littéraires.

Une illustration en serait la tendance à créer de petits « romans»; ainsi Mme de Graffigny réagira au récit fait par Devaux de son penchant pour Mme Lemire en écrivant: «Ton petit roman est le plus joli du monde, mon ami. Si tu en avois retranché tes rabachages d'excuses sur la longueur, il seroit parfait. Il m’a fait mille plaisir; celui de ta confiance est le plus vif, mais les autres se font sentir» $(3: 369$, lettre 463, 26 septembre 1742). Quelques années plus tard, les deux correspondants appelleront Saint-Lambert «Bellérophon », d'après le héros mythologique qui a tué le monstre fabuleux la Chimère, celle-ci étant une nouvelle appellation pour désigner Mme Du Châtelet (9: 227, lettre 1283,16 août 1748 et 9: 236, lettre 1288, 27 août 1748). De même, les petits contes de «Taupe Ma Mie» [Mme de Stainville] montrent l'effort délibéré de transformation littéraire du quotidien chez Mme de Graffigny $^{16}$. Un grand nombre de surnoms employés par Mme de

11. Sur la place privilégiée occupée par Mme de Sévigné dans les lettres de Mme de Graffigny, voir English Showalter, «Authorial Self-Consciousness in the Familiar Letter: The Case of Madame de Graffigny », Yale French Studies, 71, 1986, p. 113-130.

12. Heidi Bostic, "Graffigny's Self, Graffigny's Friend: Intimate Sharing in the Correspondance, 1750-52", Studies in Eighteenth-Century Culture, 42, 2013 , p. 216.

13. Elizabeth MacArthur, Extravagant Narratives: Closure and Dynamics in the Epistolary Form, Princeton, Princeton University Press, p. 159.

14. Ibid., p. 42.

15. Ibid., p. 117-120.

16. Voir Renaud Redien-Collot, «Le statut d'auteur dans la correspondance privée de Mme de Graffigny: assomption et renonciation », Studies on Voltaire and the Eighteenth Century, 12, 2004, p. 94-97. 
Graffigny continuent à offrir plaisir et piquant aux éditeurs et aux lecteurs de la Correspondance. Un jeu morphologique lui permet de créer la série «Babiole, Babiolin, Babioline, Babiolinet» ainsi que celle de «Balivernette, Balivernin, les Baliverneux » pour désigner respectivement certains membres des familles Alliot et Stainville. Ces procédés fantaisistes relèvent d'un désir de créer une société d'intimes en rendant le texte fermé aux non-initiés. Notons aussi que Mme de Graffigny elle-même aura toute une série de surnoms («Abelle, Notre Amie, la Grosse ») et qu'elle se désignera souvent ainsi en employant la troisième personne du singulier, ce qui lui permet de se transformer en personnage. Devaux sera non seulement «Panpan et Panpichon », mais aussi «Abeau, et Monsieur Je», parmi d'autres appellations. À certains moments, on semble passer bien au-delà du simple plaisir de création inventive vers une caractérisation positive ou négative des personnes désignées. Un exemple en serait le changement de surnom de Charles-Pinot Duclos, qui était d'abord surnommé «la Douceur» pour ensuite recevoir le sobriquet «la Rancune», nom d'un personnage misanthrope du Roman comique de Scarron. Une analyse selon cette perspective pourrait se faire pour bien des surnoms utilisés par Mme de Graffigny; on est même tenté de parler de défoulement dans le cas du très grand nombre de surnoms donnés à Mme Du Châtelet qui est d'abord «Dorotée», «Émilie», «la Nymphe de Cirey» pour devenir par la suite «la Bégueule », «la Chimère », «l'Ennemie », «la Mégère », et «le Monstre $»^{17}$.

Dans la Correspondance de Mme de Graffigny, les surnoms servent à renforcer l'intimité de la conversation, à se donner du plaisir (et à extérioriser des émotions) et à imiter les pratiques des gens de la République des lettres. Mais s'agit-il d'une véritable écriture codée? Comme nous l'avons déjà noté, l'emploi des adjectifs mélioratifs dans des expressions comme «le beau Ministre » pouvait offrir une mesure de protection en cas d'ouverture des lettres. Par ailleurs, nos correspondants mentionnent explicitement la nécessité de changer certains surnoms à des intervalles réguliers pour se protéger. Et il y a d'autres stratégies pour semer la confusion afin de dépister les curieux et rendre

17. À ce sujet, voir Suzan van Dijk, «Les femmes se lisaient-elles? Importance des collègues pour la venue à l'écriture», Studies on Voltaire and the Eighteenth Century, 2004, 12, p. 138-139. 
le décodage plus difficile. Par exemple, Mme Graffigny emploie un même surnom pour désigner différentes personnes: ainsi, la «Belle Dame» désigne, à différents moments, la marquise de Boufflers, la marquise de Pompadour et la marquise Du Châtelet. Et «Monsieur Je» désigne Desmarets, James Forrester, le marquis de Grandville et Devaux. Il arrive aussi qu'elle emploie le même surnom pour différentes personnes à l'intérieur d'une seule lettre (par exemple, «le Bon Ami » dans la lettre 2100, 25 janvier 1754). Une autre stratégie est d'employer différents surnoms pour désigner une seule personne, parfois à l'intérieur du même paragraphe (par exemple, «Ton Idole» et «V.» pour désigner Voltaire dans la lettre 1962, 27 décembre 1752). On peut en conclure que pour certains sujets et à certains moments critiques, l'emploi des surnoms constitue une véritable écriture codée chez Mme de Graffigny, qui choisit d'agir par prévoyance, pour se protéger ainsi que ses amis.

Quels seraient de tels sujets et de tels moments? Un premier moment critique serait la période du séjour de Mme de Graffigny à Cirey chez Voltaire et Mme Du Châtelet, visite qui s'est très mal terminée pour notre épistolière. Mme de Graffigny avait l'impression d'être espionnée; elle remarquait que d'autres ouvraient ses lettres:

J'ai recu hier, mon cher Panpan, votre lettre du trois janvier. C'est toujours un ordinaire retardé et toujours une de perdue; celle-ci a eté decachetée et tres mal recachetée. On dit que cela arrive tres comunément dans les postes quand on y voit souvent la meme ecriture et la meme forme de lettre. Je le croirois volontier, car celle que je reçu hier de Clairon n'a point eté ouverte, ny les autres qui me vienent de Luneville. Ceux qui s'amusent a nous lire perdent bien leur tems. Qu'importe? Cela est desagreable (1: 269, lettre 73, 8 janvier 1739. Voir aussi le début de la lettre 75, 12 janvier 1739).

Sans savoir que c'est Mme Du Châtelet qui ouvrait les lettres, les deux correspondants avaient déjà inventé les surnoms de «Dorotée» et « Nicodème» pour désigner la marquise et Voltaire. Évidemment un certain ton s'imposait, comme le rappelle Mme de Graffigny à Devaux: «Prenez donc garde, butort, comme vous parlez de Dorotée. Un peu plus de tournure, s'il vous plaît, prenez exemple sur la façon dont je vous en parle. Cela ne sauroit etre trop eloigné de la curiosité. Si votre letres c'etoit perdue, ce seroit un beau mistère! Quand vou[s] 
vousdré en parler, songé que tout cela se passe sous vos yeux » $(1: 236$, lettre 65, 19 ou 20 décembre 1738$)^{18}$.

Après la confrontation avec Mme Du Châtelet et le départ de Mme de Graffigny de Cirey, les deux correspondants continuent à être prudents en donnant des nouvelles de ces deux illustres personnes. Pour désigner Voltaire, il y a un nombre assez restreint de surnoms: «Atis », «Nicodème», «Ton Héros», «V.», et le plus souvent «Ton Idole» pour capter l'engouement de Devaux. Quant à Mme Du Châtelet, on trouve une douzaine de surnoms pour la désigner dont certains, comme on l'a vu, sont très révélateurs de l'inimitié de Mme de Graffigny. C'est en employant un de ces surnoms qu'elle écrira à Devaux: «Dumay est venu le soir, mais il ne nous a pas empeché de continuer. Il m'a conté que la Megere a eu un bon affront à Versailles » (6: 229, lettre 815, 3 mars 1745). De même, la prudence s'imposait lorsqu'elle donnait des nouvelles de Voltaire, surtout lors des démêlées de l'Idole avec le roi de Prusse: «Guillot [Nicolas-Claude Thiriot], le berger du vilage, est venu me voir ce matin. Il m’a conté des choses bien terrible de Ton Idole. J'avois deja vu denierement entre les mains d'un Prussien [...] une letre de V. qui se fouroit dans la dispute de Koenik contre Maupertui » (13: 148, lettre 1962, 27 décembre 1752). Et l'année suivante elle écrira: «Il est tres certain que l'ami de Guillot [Voltaire, l'ami de Thiriot] a fait les vers dont je vous ai parlé [sur Charles-Édouard Stuart le jeune Prétendant], et que c'est l'origine de tout ce qui est arrivé depuis» (13: 397, lettre 2068, 3 octobre 1753). Un autre exemple qui date des premières années de la Correspondance est celui de la grossesse d'une personne appelée «Berline-à-glace»; surnom qui permet de cacher l'identité de la princesse Anne-Charlotte de Lorraine $(1: 552$, lettre 144,16 juin 1739 et $2: 22$, lettre 150 , 30 juin 1739). Le recours aux surnoms constitue en fait une écriture codée lorsque Mme de Graffigny aborde des sujets sensibles.

Deux paragraphes pris dans une lettre de 1745 illustrent ce procédé lorsque Mme de Graffigny annonce à Devaux des nouvelles de la cour :

18. English Showalter donne un autre exemple d'écriture codée lors du séjour de Mme de Graffigny à Cirey dans son article «L'interdit dans la correspondance de Françoise de Graffigny », art. cit., p. 143-146. 
Notre Medecin [Louis XV] a enfin pris femme. [...] C'est une sousfermiere, jolie comme le jour, de l'esprit, des talens. Le St [Adhémar] la connoit sans doute; je te la nomerai quand il me plaira. [...] Aussi d'un commun accort les mariés ne se voyent-ils que deux fois la semene, le mercredi et le samedi. C'est l'encien ami du marié [le duc de Richelieu, ami de Louis XV] qui a encore la direction du ménage. [...] Le grand embaras est de faire avoir a la nouvelle marié [Mme d'Étiolles, future marquise de Pompadour] un etat qui soit plus convenable a celui de la famille du Medecin (6: 251, lettre 820, 14 mars 1745).

Une dizaine de paragraphes plus loin, dans la même lettre, Mme de Graffigny nomme la mariée: "le roi va a la comedie dans une loge grillée et y admet madame d'Etiole qui est une tres jolie femme de Paris» (13: 253). Notons les multiples précautions: des surnoms pour désigner Louis XV, Mme de Pompadour, le marquis d'Adhémar et le duc de Richelieu, ainsi que la séparation de la mention explicite du roi et de Mme d'Étiolles, comme si les deux paragraphes de cette lettre (au sujet des mariés et ensuite à propos du roi et de Mme d'Étiolles) ne portaient pas sur le même sujet.

D’autres exemples abondent dans les lettres de 1756 et 1757, période marquée par l'attentat de Damiens contre le roi, les débuts de la guerre de Sept Ans, et des conflits entre le roi et les parlements. Mme de Graffigny verra quatre de ses proches disgraciés: Helvétius lors de la publication de son livre De l'esprit, Maillebois qui sera emprisonné à la citadelle de Doullens, et les deux frères Liébault, Nicolas-François et Nicolas-Léopold, accusés d'avoir détourné des documents aux archives de la Guerre. Léopold est détenu à la Bastille en 1758, et Mme de Graffigny fait des efforts auprès des «grands » pour qu'il soit transféré à Vincennes. Elle se sert d'une écriture codée pour expliquer à Devaux ce qu'elle fait:

Tonton [Jeanne Boismont servante de Léopold Liébault] a vu Robin [Léopold Liébault] au moien d'un placet que je lui ai fait faire. Cela se fait souvent. Mais va te promener. Je ne veux pas te conter tout cela.

J'ai eté avertie qu'il ne seroit pas inpossible de faire changer de maison a Popol [Léopold Liébault], et comme je ne voudrois pas qu'il garda la siene, je vais mardi trouver quelqu'un [probablement la princesse de Talmont] a qui je dois parler pour cela (15: 270, lettre 2455,23 juillet 1758).

Notons que Mme de Graffigny utilise ici deux surnoms différents [Robin/Popol] pour désigner Léopold Liébault, qu'elle annonce le 
projet du transfert à Vincennes comme une simple histoire de changement de domicile, et qu'elle fait le sommaire de ses activités dans deux paragraphes séparés comme s'il s'agissait de deux sujets différents. Dans cet exemple, comme dans celui de la nouvelle maîtresse du roi, elle veut donner l'impression qu'il s'agit d'une histoire de ménage, ou d'affaires personnelles auxquelles les autorités auraient tendance à n'accorder aucun intérêt.

De tels exemples montrent que la prévoyance jouait un rôle certain et que par moments, l'emploi des surnoms constituait une véritable écriture codée pour dérouter les curieux et cacher la vraie identité des personnes dont on discutait. Cependant, l'emploi des surnoms est loin d'être synonyme d'écriture codée. Chez nos correspondants, les surnoms révèlent avant tout le plaisir esthétique de faire appel à l'imagination, à l'intelligence et à la culture littéraire et théâtrale, ainsi que le désir de se créer un langage privé pour renforcer et refléter l'intimité de leur amitié. Et n'oublions pas le contexte des protocoles et des conventions de l'époque. Georges Minois, dans son étude Censure et culture sous l'Ancien Régime, souligne que la censure était « une réalité fluctuante et insaisissable » et qu'elle frappait « de façon discontinue et désordonnée $»^{19}$. Il note que le pouvoir «interdit et censure officiellement et, en même temps, tolère la contestation ${ }^{20}$ ». Il y avait un réseau de complicités entre les gens de lettres et les autorités; au lieu d'assister à des affrontements, on assiste à ce que Minois appelle « un ballet tout en finesse, avec des règles tacites, implicites ${ }^{21}$ ». Dans les lettres de Mme de Graffigny, l'emploi des surnoms - qui devient au besoin une écriture codée - constitue une belle variante de ce «jeu subtil» entre les gens de lettres et les autorités.

19. Georges Minois, Censure et culture sous l'Ancien Régime, Paris, Fayard, 1995, p. $213-214$.

20. Ibid., p. 210.

21. Ibid., p. 182. 